\title{
Positioning of human resources in a construction environment using Zigbee
}

\author{
Mithun Raj, and Koshy Varghese ${ }^{*}$ \\ Department of Civil Engineering, IIT Madras, Tamil Nadu, India \\ *Corresponding author (koshy@iitm.ac.in)
}

\begin{abstract}
Purpose The purpose of this work is to explore the feasibility and accuracy with which people can be located in a construction site using Zigbee technology. Such positioning systems will enable the tracking of workers and this is critical in work areas such as tunnels and other constrained spaces which can be hazardous. Furthermore, such systems can detect worker congestion and work in unallocated areas which can lead to better planning and improved productivity. Earlier work on positioning has explored technologies such as GPS, RFID, WLAN, Bluetooth. The cost associated with most of these methods has been significant and limits widespread use within a construction site. Hence configuring a cost-effective technology was also a key requirement of this study. Method The investigation was done in three phases. In the first phase the Zigbee system was configured in a laboratory environment and accuracy of positioning was established. In the next phase, the system was mounted on a construction helmet and tests were conducted within enclosed spaces of a construction site. In the final phase the system mounted on a helmet was worn by a worker and tested outdoors on a construction site. In all phases the factors affecting the position accuracy were analyzed. Results \& Discussion The seven positions identified in the table of the final phase of investigation correspond to the division of the site into grids. The total number of positions evaluated and the number of these positions which were correctly classified are specified in the table. It can be seen that the accuracy of the classification ranged from $74.3 \%$ to $93.3 \%$. It was found that the accuracy varied based on the distance of the position from transmitters as well as proximity of the position to the edge of adjacent grids. Based on these investigation guidelines on the configuration and usage of Zigbee based networks for positioning construction personnel were developed. In addition to the technological feasibility a cost comparison was also done and it was found that the Zigbee technology could be implemented at about 1/10th of the cost of WLAN and other technologies. Although this technology was only tested on construction workers it is directly applicable for location of other subjects such as differently abled or elderly persons whose location needs to be monitored remotely.
\end{abstract}

Keywords: automation, positioning, Zigbee

\section{INTRODUCTION}

Construction is one of the largest and fast developing industries in Indian subcontinent. When the level of automation of construction in India is compared with developed countries, it is apparently much less and needs significant improvement to ensure pace of development is maintained. Application of software like Primavera and Microsoft project have already been in place and play an important role in planning and scheduling of most construction projects. Automated data collection \& sensing technologies like RFID (Radio Frequency Identification), WLAN (Wireless Local Area Network), GPS (Global Positioning System), WSN (Wireless Sensor Network) etc. have gained importance in recent times, however applications are very limited.

Positioning of resources in indoor environment always draws attention as it can significantly help in safety and risk management, asset management and maintenance, productivity and time management. Research on wireless positioning systems have employed Ascension technologym ${ }^{1}$, Differential GPS (DGPS), Wireless assisted GPS, Ultra Wide
Band (UWB), Proprietary microwave solutions, Wireless LAN (WLAN) ${ }^{2}$, Bluetooth, Infrared, Radio Frequency Identification (RFID), Ultrasonic, GSM and CDMA mobile technologies. Wireless technologies gained importance in customer friendly application and helped in resolving various problems in the field of medical, industrial, public safety, logistics and transport systems. . The process of finding location information using wireless technologies is called location sensing, geo-location or radiolocation. This study introduces an indoor object tracking method using Zigbee module under IEEE 802.15.4 ${ }^{3}$ which gives meter level accuracy.

Automatic location can be applied in many real world applications. Location detection of warehouse products, tracking of hospital personnel as well as patients, firemen detection in a building on fire, detection of police dogs trained to find explosives in a building, tracking maintenance tools and equipment's and tracking of pipe spools and other spares in a large manufacturing plant. Management of materials, machinery and vehicles can be controlled by computers and drivers whereas control of labor is much more difficult and hence requires more attention. 
Indian construction industry is labor driven and management of labor forms an integral part of major projects. Tracking of labor in indoor environment has always posed problems as there is no proper automated technique in construction environment particularly in the Indian context. The labor working inside tunnels, congested areas and similar environments have to be tracked so that there should be clear idea about the number of labors employed inside these environments in case of an emergency. The conventional system of written entry of in and out time in register has many limitations.

Another important issue is productivity of labor employed in indoor environment. From field visits, it was observed that most of the labors employed inside the tunnel or indoor environment are idle for significant amount of time and there was no effective monitoring. The same has been observed in congested indoor environments of nuclear power plants and it was impossible to know how many labors are working in a particular area. Hence an automated system to monitor the movement of labor is essential. Through such a system, positioning and tracking of labor, the number of workers, proximity to hazards and state of labor i.e. whether a labor is static or dynamic can be determined and appropriate action can be taken based on the situation.

As such systems have the potential for widespread use it has to be economical to implement and give reasonably good results. The objective of this study was to explore various technologies which can be used to implement such a positioning system and develop a cost-effective prototype implementation for an appropriate technology.

The next section of this paper reviews factors influencing positioning and various positioning technology. This is followed by system architecture of a Zigbee based technology. The calibration, implementation and testing details of the technology are presented next and finally the conclusions of the study are discussed.

\section{Classification of Positioning Systems}

Liu et al. (2007) $)^{4}$ and Zhang et al. (2010) $)^{5}$ classified the various positioning systems used in the industry. Positioning of one's own location has always gained importance since early times. A study on the constellation of celestial bodies was also a tool for knowing one's own location. Achievements in mathematics and hence the laws of trigonometry, along with inventions of novel measuring instruments lead to the development of many positioning techniques. Principle of localization follows the same principle as in the ancient times. The constellation of stars used to deduce the location is now replaced by reference points with respect to which the location is tracked. Similarly positioning can be also classified into client based, network based and network assisted (Alex Varshavsky $(2009)^{6}$.Client based positioning does not depend on the network infrastructure to track themselves, whereas the second one employs the network infrastructure to calculate the location of the object. The network assisted incorporates the facilities of both to get the location of the mobile user.

The selection of a positioning technique depends on various factors. Even though there are many indoor positioning techniques, there is no single technique which can satisfy the entire criterion. A positioning method which is apt for one use may not be suitable for another purpose and hence should be selected wisely.

\section{Parameters influencing selection of techniques}

The significant parameters which decide the selection of a particular technique are listed below:

\section{Accuracy}

One of the most important criteria for selection of a technique is accuracy. Accuracy can vary from millimeter range to kilometer range depending on the technology used. As the accuracy increases, performance of the system is considered to be better. Accuracy can be used as a main parameter for biasing the selection of a positioning technique.

\section{Precision}

Precision is the measure of robustness of a positioning system. The ability of a positioning system to reproduce the same results over multiple trials reveals the precision of a tracking system. Accuracy gives the error whereas the precision gives the consistency of a system.

\section{Complexity and Responsiveness}

The complexity of location system refers to complexness of the hardware, software and operation factors involved in implementing a particular system. The complexity of a system is indicated by location rate. The delay between target moving to a new location and system reporting the new target location gives the lag which in turns the gives the location rate.

\section{Robustness}

The ability of a positioning system to work even in the harsh and unexpected condition is referred to as the robustness of the system. For example suppose a system is not receiving signals from all the sources and if the system is able to give the location of target by processing existing or available signals, and then the system is referred to as robust. 


\section{Scalability}

The extent of coverage of a positioning system in terms of both area and density is known as the scalability of a tracking system. The system should ensure proper working even if the scope gets widened to a reasonable limit. Another measure of scalability is the dimensional factor of the system, whether an object can be located in $2 \mathrm{D}$ or $3 \mathrm{D}$.

\section{Cost}

Cost of a system involves many factors which include money, time, space, weight and energy. Time of installation and maintenance is the time factor. The system may have tight space and weight constraints. Usage of passive energy or active energy sources have a significant effect on the system and hence the energy factor.

\section{Power Consumption}

The usage of power should also be considered especially when battery driven mobile units are involved.

\section{Compatibility}

The selected technology should be compatible with the working labors and also with the human beings who live within the range of the positioning system.

Khoury \& Kamat $(2009)^{7}$ studied the use of WLAN (Wireless Local Area Network) technique for user localization in indoor construction environment. Wireless positioning systems can be classified based on different measurement techniques adopted to position the location of Mobile Station (MS). The common measurement techniques used today are Trilateration, Triangulation, Proximity, hyperbolic lateration, Fingerprinting and Dead reckoning techniques. The trilateration method computes the location by measuring the distance between fixed reference nodes of known location and the MS. The technique adopts the principle of Time Of Arrival (TOA) and signal strength attenuation for the calculation of distance.

The method of triangulation uses the principle of AOA (Angle of Arrival) for the positioning of MS. The angle of arrival of radio signals is obtained to derive the location of MS. Directional antennas are used for obtaining AOA. The point of intersection of two angular rays are sufficient for locating the position of MS in 2-D; but in practice, more than two reference nodes are considered in order to reduce the errors in angular measurement. When trilateration uses the TOA (Time of Arrival) for location determination, hyperbolic lateration uses the TDOA (Time Difference oif Arrival) for estimating the position of MS. The difference between signal arrival times at two reference points gives the location of MS on a hy- perbolic line where the reference nodes form the foci. Few examples of system that uses TOF (Time of Flight), AOA and TDOA are Cricket ${ }^{8}$, Active bat, Ubisense, Sapphire darts etc.

Fingerprinting $^{9}$ or location pattern matching technique uses the measurement of RSS and its unique behaviour in a particular location forms the basis of the method. In this method the location of the MS is calculated by finding the closest match from the fingerprint database of the area. The disadvantage of using this method is the factor of time as it consumes lots of time for creating the RSS database. The advantage of the system is the fact that MS does not have to search for the signals from all the three reference nodes for location tracing as this technique depends only on the pattern matching.

Proximity is another method for localization in which closeness of an MS to a particular reference node is used to find the location of the user. It can be direct contact or even the presence detected by sensors at reference nodes. This method may not reveal the identity of the MS, for that additional arrangement should be incorporated. Some of the examples include active floor $^{10}$ which uses step up sensors for location identification by direct contact, active badge $^{11}$ using infrared, airbus ${ }^{12}$ which works on the principle of differential air pressure, camera, Bluetooth $^{13}$, RFID $^{14}$ and cell of origin method using cell phones.

Dead reckoning is the method of positioning by calculating from the known previous location of the MS, and its direction of travel, speed and elapsed time. This method is basically on the assumption that the direction and the average speed of travel of MS from the last known location is either known or can be estimated. The commonly used sensors for dead reckoning technique are accelerometers for measuring the acceleration, odometers for measuring the distance travelled, and gyroscopes for assessing the direction of travel.

The positioning systems are again classified on the basis of different methods or systems adopted for localizing. The wireless methods commonly used for location tracking are Radio frequency $\left(\operatorname{RADAR}^{15}\right.$, SPOT ON ${ }^{16)}$, Infrared, Bluetooth, Ultrasound, Inertial navigation systems and sensors. The signal characteristics like propagation delay, reflection, scattering and diffraction affect all the above mentioned technologies. The other factors like range, available bandwidth, regulatory constraints, interference, power constraints, safety and cost of the technology can also influence the selection of the suitable technology.

\section{Wireless Sensor Networks (WSN)}

WSN uses accurate and low cost sensors for localization of MS. The sensors work on IEEE 802.15.4 
infrastructure and operate at a frequency of $2.4 \mathrm{GHz}$. The two major sensor network standards are IEEE 802.15.4 PHY (PHYsical Layer) layer and MAC (Medium Access Control) layer for LR WPAN (Low Rate Wireless Personal Assisted Network) and Zigbee networking ${ }^{17}$ and application standards. The Zigbee protocols use RSS for the localization purpose. The thesis work is based on localization system based on Zigbee protocol using RSS. Fingerprinting technique is adopted and Euclidean Distance algorithm is considered for tracking the MS.

Bob Heile, The chairman of Zigbee Alliance defines ZigBee as "a rapidly growing, worldwide, non-profit industry consortium" whose mission is "to define a reliable, cost-effective, low-power, wirelessly networked, monitoring and control product based on an open global standard." ${ }^{18}$ It also has the following features.

- $\quad$ Low power consumption compared to Wi-Fi and Bluetooth

- Doesn't require any extra infrastructure

- Cost effective

- Works without internet access

- Mesh Self-Healing network

- $\quad$ Good range (10m to $100 \mathrm{~m})$

Various technologies were considered for the study and finally Zigbee protocol was selected for localization. The comparison between different technologies considered during the initial study is given in Table 1.

Table.1. Cost comparison between different tracking technologies

\begin{tabular}{|c|c|c|c|c|c|}
\hline $\begin{array}{l}\text { Technologyl } \\
\text { Module }\end{array}$ & Vendor & Infrastructure & Range & Cost & $\begin{array}{c}\text { Total cost } \\
\text { unit }\end{array}$ \\
\hline \multirow{2}{*}{ RFID } & Gao RFID & $\begin{array}{l}\text { RFID reader } \\
\text { RFID tag }\end{array}$ & $5 m-10 m$ & $\begin{array}{c}45000 \\
1800\end{array}$ & 46800 \\
\hline & Barcode India & $\begin{array}{l}\text { RFID reader } \\
\text { RFID tag }\end{array}$ & $7 \mathrm{~m}$ & $\begin{array}{c}55000 \\
1500\end{array}$ & 56500 \\
\hline \multirow[b]{2}{*}{ Wi Fl } & Aeroscout & $\begin{array}{l}\text { Reader and softwares } \\
\text { Tag }\end{array}$ & $10-100 \mathrm{~m}$ & $\begin{array}{c}75000 \\
3000\end{array}$ & 78000 \\
\hline & Wherenet & $\begin{array}{l}\text { Cisco CCX - LWAP (light } \\
\text { weight access point) } \\
\text { Cisco Location Appliance } \\
\text { also known as an MSE } \\
\text { Location switches from } \\
\text { Cisco are necessary } \\
\text { A wireless LAN controller is } \\
\text { needed } \\
\text { Wherenet tag }\end{array}$ & $10-100 \mathrm{~m}$ & 100000 & 102500 \\
\hline WSN - RFID & Rasilant Tech. & $\begin{array}{l}\text { Active RFID tag, Wireless } \\
\text { sensors }\end{array}$ & $10-30 \mathrm{~m}$ & 350000 & 350000 \\
\hline Zigbee & Maxstream Xbee & \begin{tabular}{|l} 
Xbee modules \\
Arduino UNO \\
Switching board
\end{tabular} & $10-100 \mathrm{~m}$ & $\begin{array}{l}1500 \\
1800 \\
2500\end{array}$ & 5800 \\
\hline
\end{tabular}

In this study, the feasibility of using Zigbee 802.15.4 protocol is evaluated for positioning of human resources and the effectiveness of Zigbee based tracking system is studied in actual construction site, both in indoor and outdoor environments. The work focused mainly on the use of Zigbee Maxstream $\mathrm{Xbee}^{2}$ module of IEEE 802.15.4 standard for location estimation. The study also focused on the use of Audrino $\mathrm{UNO}^{3} \mathrm{MCU}$ platform and its implementation for the object tracking application. The research used fingerprinting technique for location estimation.

The primary step of the research was to select appropriate technology and algorithm for location estimation through literature review of various positioning technologies. Zigbee protocol (802.15.4) was selected for the study and Maxstream Xbee module was selected as the module for location estimation system. The next step in the research was to study about Xbee module and Arduino UNO board through appropriate literature review. The selected modules were then configured for tapping RSSI (Received Signal Strength Intensity) using various command modes. Fingerprint models were then created and location identification system was developed on the basis of developed models. The location estimation system was then experimented for its performance parameters through various tests conducted at actual construction sites.

\section{LOCATION ESTIMATION USING ZIGBEE}

\section{RSSI Mapping}

The Indoor localization of human resources using Zigbee is based on location fingerprinting technique. Kaemarungsi (2005) has studied the use of location fingerprint technique. A fingerprint based on RF is created to represent a particular location. RSSI of various RF waves are used for creating a location fingerprint. The basic assumption behind the creation of a location fingerprint is uniqueness of RF signature at a particular location inside the building. The RSS pattern at a particular location inside the building is assumed to be unique and hence an authentication is created. The fingerprint is usually designated along with the location information. The fingerprints along with their location information are maintained in a database and the same is referred during the tracking or online phase to estimate the location. The location information about an indoor location can be stored in two forms; either in the form of coordinates or as a location indicator. The indicator in coordinate system may have one dimension to five dimensions where the five dimensions include three dimension space and two orientations. A location information in two dimension with orientation can be expressed as $\mathrm{L}=\left\{(\mathrm{x}, \mathrm{y}, \mathrm{d}) \mid \mathrm{x}, \mathrm{y} \quad \mathrm{R}^{2}, \mathrm{~d} \quad\right.$ NNorth, East, South, West $\}\}^{19}$.

RSS is found to fluctuate lots with time for each access point and location. The behaviour of RSS is highly variable, hence needs to be studied for better results. The behaviour of RSS can be observed by recording its static behaviour, approximating its distribution or by maintaining the whole dataset. The pattern of RSS and its analysis is required for the selection of suitable location estimating algorithm. The location fingerprint is stored as RSSI values in 
the form of arrays and the size of the array depends on the number of access points that can be heard. Static analysis of RSS data is called pre-processing and is considered important. The pre-processing refers to the cleaning of raw data. Cleaning may consist of encoding, noise reduction, feature extraction/selection etc.

To create a basis fingerprint, a set of RSSI values is collected for a period of time from all the access points at a particular location. This basis is called a prototype. Then, the average RSS of each access point is calculated and recorded as an element in the location fingerprint. For an area that can receive signals from $\mathrm{N}$ access points, the location fingerprint can be expressed as a vector of average RSS elements $\beta \mathrm{i}$ :

$$
F=\left(\beta_{1}, \beta_{2} \ldots \beta_{N}\right)^{\top}
$$

This location fingerprinting technique uses the authentication of measured RSSI to estimate the location of the mobile station. By knowing RSSI at known locations and the instantaneous RSSI, the position is estimated. The RSSI at known locations should be collected and stored at the central database as Radio map or Radio signatures. This type of positioning systems does not require any extra infrastructure as it depends only on the capability to display the RSSI. Hence it is simple to deploy this technique compared to other techniques. Indoor positioning methods also encounter the problem of NLOS (Non Line Of Sight) and hence the method of AOA (Angle of Arrival) and TDOA (Time Difference of Arrival) becomes less applicable in indoor environments.

\section{Finger printing Technique}

The Fingerprinting was implemented in two phases: The training phase and the tracking phase. In the training phase, a 2-D virtual map is created and the area of interest was divided into grids. The entire area of scope should be covered and reference points should be evenly distributed. Mobile user with active tag is made to stand in all the reference points and corresponding RSSI from the tag is noted from the access point. With all the RSS obtained, a database of RSS is created which is called the Radio Map. Objective of the training phase is to create a signal information database. It acts as a base or underlying reference for the tracking phase. The RSS obtained might have lots of interference with noise signals and all those disturbances should be cleared or leveled. The dataflow in training phase is shown in Figure 1.

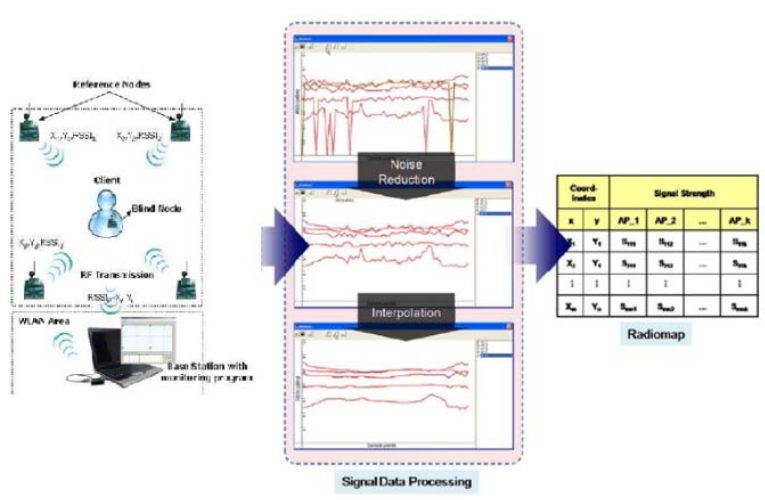

Fig.1. Dataflow of Training phase

In the second phase, a MS (Mobile Station) will report a sample RSSI from different access points and the same is send to the base station. The location of MS is estimated at the base station using proper algorithm. The most commonly used algorithm for location estimation is Euclidean distance algorithm ${ }^{20}$. It computes Euclidean distance between reported RSSI and RSSI's at each point in the Radiomap and returns that location with the smallest Euclidean distance. A good database should be created with proper planning and system design. If location information is not accurate, creation of more fingerprint locations are preferred. Factors such as number of access points and grid spacing should be decided on the basis of system performance. Parameters that improve the accuracy and precision to a greater extent are still not clear. The dataflow in training phase is shown in Figure 2.

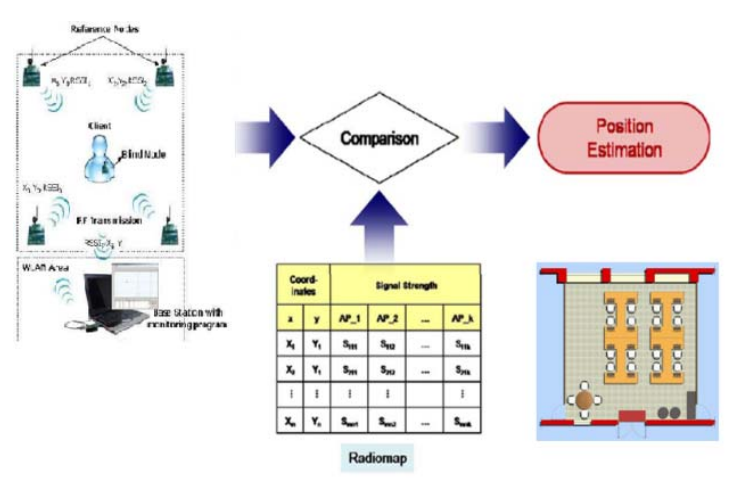

Fig.2. Dataflow of tracking phase

\section{Location estimation}

To estimate the location, an algorithm is created called as location estimation algorithm or positioning algorithm which exploits the relation between location information and location fingerprint in order to determine the location from sample of RSS signals. Euclidean Distance algorithm is a method of location estimation in which a set of constant fingerprints which includes mean RSS vectors are used. The set of mean RSS vectors are collected during the offline phase and stored to represent a particular location. The RSS vectors obtained during the online phase is 
then mapped to previously stored fingerprint vector and mapping is then converted into position of the user. The Euclidean distance algorithm calculates the distance between the online phase RSSI vectors and the offline RSSI vectors and the returns the position having the smallest distance or least error as the location of the user.

Location estimation using Zigbee protocol was carried out using Maxstream Xbee module, Arduino UNO micro controller boards and switching boards to act as a platform for connecting Xbee and Arduino. The Xbee module was configured in API mode and API operation requires that communication with the module be done through a structured interface. The API command mode in the Xbee module has a definite framed structure for transmitting and receiving the RF packets. The serial data transfer is included in the frame work of API mode and hence there is no need of switching between data mode and command mode. The major limitation of switching time in AT command mode is hence removed in the API command mode. The configuration and burning of data into Xbee modules were done using X-CTU software which is open source software for Xbee. The Xbee is connected to the system in which X-CTU is installed and the program is executed. The first step before writing data is to check and establish the connection with the modem. The connection is tested in the PC setting tab of the X-CTU window. The API command mode can be directly burned into the Xbee module using X-CTU interface. The modem configuration tab of X-CTU was used to set the API mode. The power level for transmission of RF packet can also be set in the X-CTU window.

Arduino Uno MCU was configured to send and receive the packets of RSSI information from all the nodes. The program for sending and receiving the information packets was written and it was burned on Arduino MCU using Arduino 0022 software. Arduino 0022 is open source software which can be downloaded from internet and it acts as an interface for burning data into MCU board. The programming code was written and saved in ".pde" format and was embedded into the Arduino UNO board using the Arduino 0022 interface. Coding included the ND (Node Discovery) AT command and was executed for sending and receiving of packets. Arduino board on the remote node sends the ND command to all the reference nodes in the range and these reference nodes receives the command and returns the information back to the remote node. The remote node then extracts the RSSI and node information from the packets and sends them back to the base station. Figure 3 shows the data flow executed in the Arduino UNO MCU.

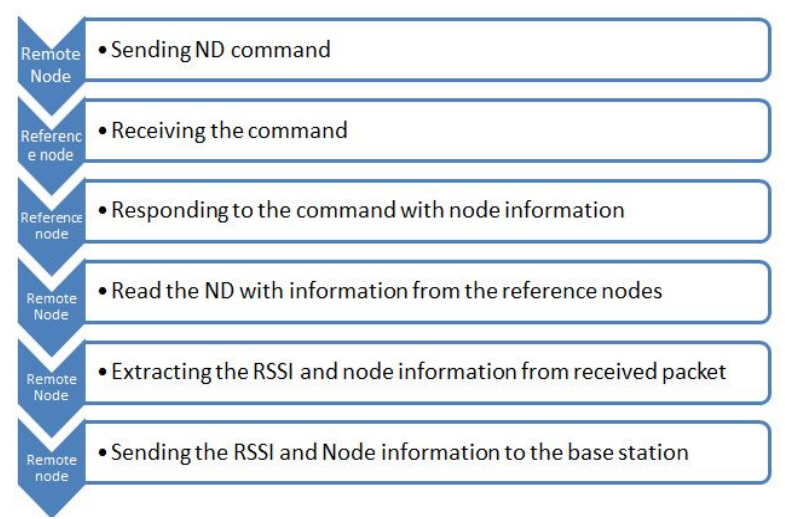

Fig.3. Flow chart showing the steps executed at Arduino Uno board

The application for object tracking is coded in Matlab and the coded program has the capability of receiving data from the modules and also to return the location of mobile node. The object tracking algorithm makes use of the Arduino platform for location estimation application.

\section{Indoor testing of zigbee}

The calibration experiment was conducted room no. 209 at Building Sciences Block (BSB) in IIT Madras. The experimental test bed is shown in Figure 4. An laptop was used as the Base Station (BS) for the experiment. The Arduino Uno MCU was connected to all the Zigbee nodes and a programmed Zigbee was connected to the laptop which acted as the BS. The configured Zigbees were used to collect RSSI form all the nodes. The Software was designed only to monitor the RF from 802.15.4 radios propagating at a frequency of $2.4 \mathrm{GHz}$. The test bed illustrated in the figure is a 3-D structure of dimension $9 \mathrm{~m}(L) X$ $6.6 \mathrm{~m}(\mathrm{~B}) \times 6.6 \mathrm{~m}(\mathrm{~B}) \times 3.5 \mathrm{~m}(\mathrm{H})$. The locations where the Fingerprints are taken are indicated in the same figure. The centerpiece of our infrastructure is the Arduino Uno platform with AT Mega 328 microprocessor and IEEE 802.15.4 compliant Maxstream Xbee Zigbee radios.

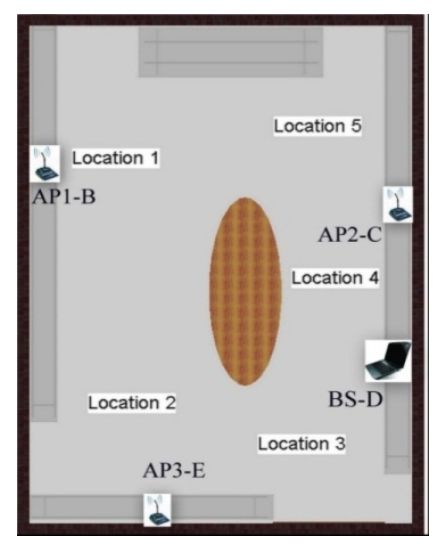

Fig.4. Plan of Test Bed for the indoor tracking 
The creation of Radiomap is the basis of Fingerprinting technique. Three reference nodes, one mobile node and a base station node were used for the creation of the Radiomap. The Base station node was connected to a Dell laptop and the data obtained was transferred to the computer. The Radiomap was created by executing the commands in the terminal of Linux operating system. The Mobile node was used to obtain the RSSI from all the three reference nodes and the same will be transferred to the base station node. The room was divided into a predefined number of grids and the mobile node was kept at all the grid locations to obtain the RSSI pattern at that particular location. A total of sixty to seventy readings were taken at each grid location. The RSSI values collected at the various locations was transferred and stored in the BS computer.

The most important step in the object tracking application is collection of data from all the sensors and it's processing at the base station. The data obtained at the base station node is transferred to the system which runs the object tracking application. When the data arrives at the serial port, the application must capture it. First the mobile node transmits the beacons and the same will be received at the reference nodes. The reference node transmits the packet containing the RSSI values. The mobile node will send all the RSSI values to base station node which is then transferred to the BS system. The object tracking algorithm then calculates the position and returns it.

The testing of the module for location tracking was conducted in room no 209 of BSB and performance was noted. Three access points AP, AP2, AP3 were kept at three corners of the Room No 209 and the base station along with the BS node is kept at the centre of the room. The mobile node is made to move randomly inside the room and the beacons are emitted continuously. The emitted beacons are then received by the fixed nodes and the RSSI values are then returned to the BS. The BS calculates the location by comparing the RSSI database or the Radiomap with RSSI values transferred by the mobile node. The location tracking application developed in the work obtains the RSSI values from all the modules and then compares with Radiomap of the area to calculate the Euclidean distance. The application returns location of the mobile station and displays the location on a Graphical User Interface (GUI).The application was coded in Matlab and the GUI image used was the JPEG image of the room plan. The accuracy of the tracked location varies from three metres to five metres. The steps involved the object tracking algorithm is shown in the Figure 5.

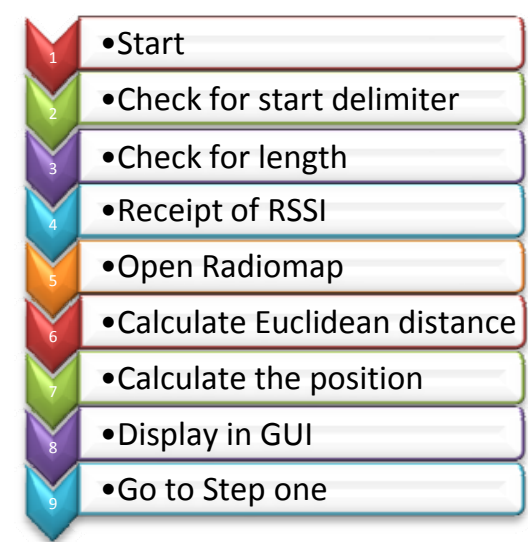

Fig.5. Steps involved in the object tracking algorithm

The algorithm calculates the Euclidean distance between the received RSSI packet and the radiomap already stored or incorporated in the algorithm. The least distance or the location with the least error was returned as the location of the mobile user. The graphical user interface was created using a bitmap image of the test bed i.e. room no 209 of BSB at IIT Madras. The GUI was created in Matlab and was incorporated in the algorithm for location tracking. The position estimated was directly switched to display the location on the map. The five locations in the radiomap were directly linked to five cases in the GUI. Each case corresponds to the display of that particular position or location to which it is linked. Figure 6 shows the display of positions in a GUI when the algorithm is executed.

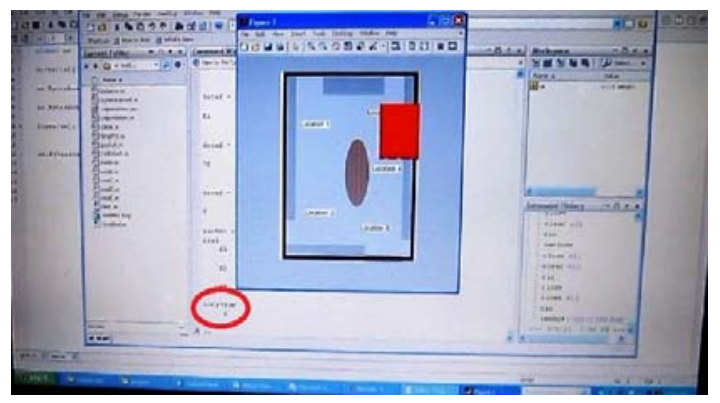

Fig.6. Graphical User Interface showing the location of the mobile user

\section{Outdoor validation of zigbee}

The acceptable performance of Zigbee in indoor environment led to testing its performance in outdoor environments. Performance of the modules were observed and analyzed at the outdoor space and it was compared with the ZigBee performance in indoor environments. The behaviour of RSSI in outdoor environment was predicted to be more satisfactory than the indoor environment due to the reduction in interference factor and less multipath effect. Outdoor analysis of Zigbee and its tracking system was experimented at a construction site inside IIT Madras. The reinforcement yard area was selected as test bed for study. The selected area was of di- 
mension $27 \mathrm{~m} \times 17 \mathrm{~m}$ with a rebar bending bench at the centre of selected area. The reference nodes were fixed at three corners of the selected test bed. The base station node was connected to an Acer laptop. All three reference nodes were battery powered due to the absence of a permanent DC supply at the site. The mobile node was fixed on the helmet and was battery powered. Figure 7 shows the helmet mounted with mobile Zigbee node.
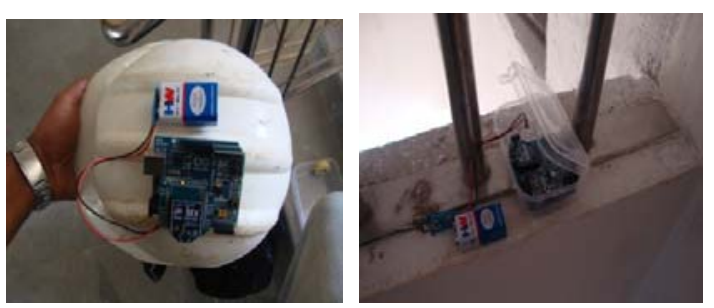

Fig.7. The experimental set up of refernce nodes and mobile node

Radiomap for the selected test bed was obtained by collecting fingerprints of the area at selected locations. Seven locations were identified for taking the fingerprints. The seven locations cover the entire test bed so that movement of remote node inside any location of test bed can be tracked. RSSI's required for creating fingerprints were obtained at all the seven locations and results were studied and analyzed. It was observed that the radio maps created for location estimation were exhibiting different patterns at different fingerprint locations. Therefore there was a difference in pattern of signatures created for outdoor tracking. Performance of reference nodes in sending RSSI values were observed at each fingerprint locations. Locations at which reference nodes displayed unacceptable behaviour were rejected and new location was selected for radiomap. Final locations which were selected for the creation of fingerprints are shown in the Figure 8. Mobile user with attached module was allowed to navigate inside the test bed and location estimation algorithm was executed. Movement of the remote node was successfully tracked by this application

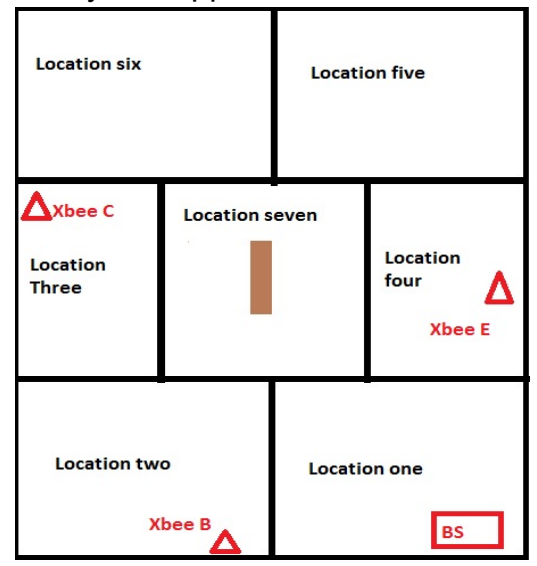

Fig.8. Fingerprint locations (GUI) for location estimation at outdoor space
The system displayed good performance in tracking of mobile user in outdoor environment. Fluctuations in location estimation were observed to be less compared to indoor location tracking. Figure 9 displays location of mobile node when location estimation algorithm was executed.

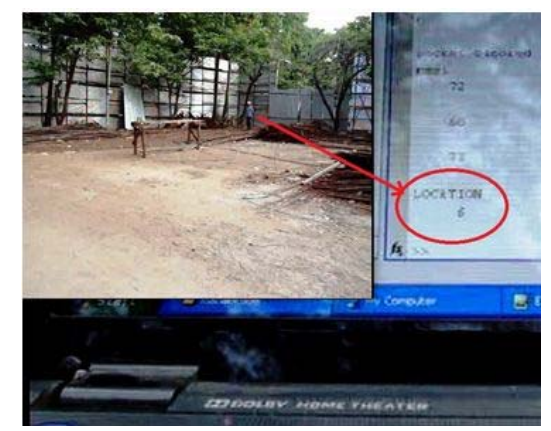

Fig.9. Test bed for the outdoor experimentation of Zigbee tracking system

Zigbee based location tracking system was tested for its performance by tracking the location of mobile user inside selected test bed. Mobile user with Xbee node was set up at various predefined positions and performance of location estimation system was analyzed. Initially the entire test bed was divided into seven grids and fingerprint location for each grid was selected as the centre of the grid. Radiomap for the entire area was created and embedded inside the location estimation algorithm. The mobile node was then allowed to move and the developed location estimation system was tested for its performance of tracking mobile node when it moves from one location to another. Accuracy and consistency of system in tracking position were tested through the experiment. Mobile node was given a pause in movement at every one metre from each fingerprinting location and position displayed by location estimating algorithm was observed. Entire test bed was tested with mobile node for studying the performance of Xbee tracking system. From performance tests, it was inferred that location estimation system developed on the basis of Zigbee protocol was having an accuracy of $3 \mathrm{~m}$ to $5 \mathrm{~m}$. Figure 10 displays the points where the system behaved erroneous.

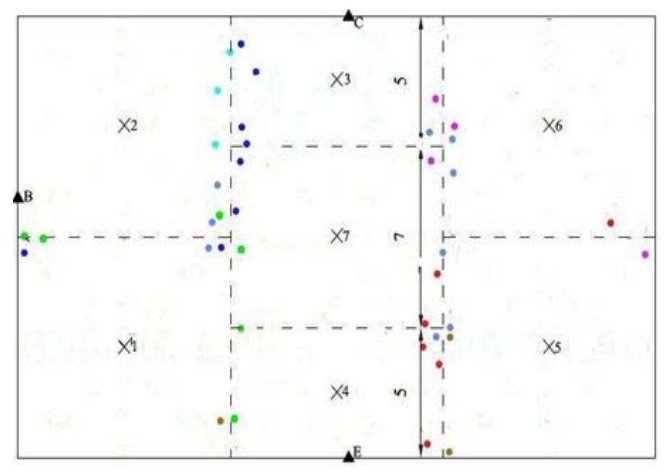

Fig.10. Actual location Vs Fingerprinting location error distribution for entire test bed 
A total of 217 points were selected for testing of location estimating system out of which 178 points displayed correct positions. There were only 39 erroneous points in the entire set. From the study, it was observed that location estimating system developed on the basis of Zigbee displayed an accuracy of 82 $\%$. The accuracy of $82 \%$ was acceptable and test proved successful. Table 2 displays total points selected for each position and error at each location. It was also observed that the system took some time to stabilize and update its new location, when it moved from one location to another. Time gap for location updating was observed to be $15-20$ seconds.

Location estimation application in this work was limited to small test bed and network. System developed for this project was designed for future expansion of the tracking network. Mobile node used for this project was programmed to consider all the nodes in the range. ATND command coded in mobile node was capable of obtaining information from all nodes available in its range. Arduino sends the received information from all nodes to the base station in a separate packet containing node information and RSSI. Packet send by mobile node specifies the length of entire packet and this length forms the check for location estimation algorithm. When algorithm is triggered for location estimation, it checks for RSSI from all nodes available in the network. Positioning will be carried out only if length of packet received from mobile node matches with number of RSSI values processed inside location estimating algorithm. The algorithm is already designed for accommodating additional nodes and hence there is significant potential for expansion of network to a large test bed.

Table.2. Accuracy of system at test locations in actual construction site

\begin{tabular}{|l|c|c|c|c|c|}
\hline Location & $\begin{array}{c}\text { Total } \\
\text { points }\end{array}$ & $\begin{array}{c}\text { Correct } \\
\text { result }\end{array}$ & $\begin{array}{c}\text { Erroneous } \\
\text { results }\end{array}$ & $\begin{array}{c}\% \\
\text { error }\end{array}$ & Accuracy \\
\hline Position 1 & 32 & 26 & 6 & $19 \%$ & $81 \%$ \\
\hline Position 2 & 35 & 26 & 9 & $26 \%$ & $74 \%$ \\
\hline Position 3 & 26 & 21 & 5 & $19 \%$ & $81 \%$ \\
\hline Position 4 & 28 & 24 & 4 & $14 \%$ & $86 \%$ \\
\hline Position 5 & 33 & 27 & 6 & $18 \%$ & $82 \%$ \\
\hline Position 6 & 30 & 28 & 2 & $7 \%$ & $93 \%$ \\
\hline Position 7 & 33 & 26 & 7 & $21 \%$ & $79 \%$ \\
\hline $\begin{array}{c}\text { Overall } \\
\text { accuracy }\end{array}$ & 217 & 178 & 39 & $\mathbf{1 8 \%}$ & $\mathbf{8 2 \%}$ \\
\hline
\end{tabular}

The tracking system developed on the basis of Zigbee exhibited good performance in tracking position of mobile node inside room no. 209 of BSB, IIT Madras. The location was estimated with an accuracy of 3-5 $\mathrm{m}$ and GUI displayed position of the mobile user on the map. System exhibited fluctuations in the position of the user and location display fluctuated in the map of GUI. Close spacing of the fingerprints, interference and multipath effect may be reasons for the fluctuation in location estimation. The performance of the system was studied in actual construction site and analysis was done. From site validation of developed tracking tool, it was observed that positioning system based on Zigbee protocol have significant potential for application in both inside and outside environment. Zigbee based system can be a better low cost tracking tool for positioning of labors in areas where GPS does not work. System showed acceptable accuracy in both indoor and outdoor space with comparatively more precision at outdoor environments. Scalability of ZigBee networks was also checked and proved to be successful.

\section{SUMmARY AND CONCLUSIONS}

The research on positioning of human resources using Zigbee networks lead to following conclusions

- From the study, it can be observed that Zigbee protocol on IEEE 802.15.4 infrastructure has significant potential in tracking of labors. The location estimating system developed on Zigbee protocol exhibited good performance in tracking of labors in both inside and outside environments. Hence it can be concluded that Zigbee has potential scope in the area of positioning of labor and construction resources.

- Due to the ability of Zigbee modules in tapping RSSI from sending and receiving packets, it was observed that fingerprinting technique based on RSSI was most suitable for the study. It was observed that the Xbee modules based on Zigbee protocol was successful in tapping RSSI from different nodes without much variation. Hence it can be concluded that location tracking using Xbee module and Fingerprinting technique is very effective and exhibits acceptable accuracy. Location tracking using fingerprinting technique displayed good and acceptable results without complex infrastructure and costly equipment's.

- The study conducted in actual construction sites proved satisfactory and exhibited good accuracy. Results from various experiments proved that system is effective in both indoor and outdoor environments. It was observed that the accuracy 
of developed location estimation system was 82 $\%$ and is acceptable.

The research in this thesis provides a basis for ground work for the future study of efficient design of positioning systems using wireless sensor networks based on Zigbee protocols. The RSSI based location estimation systems has limitations. The large variation of RSSI with time and material movement has a significant impact on positioning and this should be taken into consideration. The fluctuation of estimated location due to the variation of RSSI and its control using smoothening and filtering algorithms may reduce the large variation in RSSI. The power consumption of the modules is still an issue and research can be carried out to minimize the power consumption. The network and test area considered in this work were confined. The network can be expanded to a large area test bed and its effectiveness in productivity analysis can be studied.

\section{References}

1. Ascension Technology Corporation, "Tracking 3D world”, www.ascension-tech.com, Oct 24, 2010.

2. Maxstream Xbee, "Parts", http://ssdl.stanford.edu/ssdl /images/stories /AA236/0708A/ Lab/Rover/ Parts/ xbeeproproductmanual.pdf, Jan 5, 2011

3. Arduino, "Arduino Uno", http://arduino.cc/en/Main/ ArduinoBoardUno, Jan. 23, 2011.

4. Liu, H., Darabi, H., Banerjee, P., Liu, J. "Survey of wireless indoor positioning techniques and systems.", IEEE Transactions on Systems, Man, and Cybernetics, Part C: Applications and Reviews, vol. 37(6), pp. 1067-1080, 2007.

5. Zhang, D., Xia, F., Yang, Z., Yao, L., Zhao, W., "Localization technologies for indoor human tracking.", 5th International Conference on Future Information Technology (FutureTech), pp. 1-6, 2010.

6. Varshavsky, A., Patel, S., "Ubiquitous Computing Fundamentals" pp. 285-320, 2009.

7. Khoury, H. M., Kamat, V. R., "Evaluation of position tracking technologies for user localization in indoor construction environments.", Automation in Construction, vol. 18(4), pp. 444-457, 2009.

8. Priyantha, N.B., Chakraborty, A., Balakrishnan, H., "The Cricket location-support system", Proceedings of MOBICOM, pp. 32-43, 2000.

9. Kaemarungsi, K. "Efficient design of indoor positioning systems based on location fingerprinting," Wireless Networks, Communications and Mobile Computing, 2005 International Conference on , vol.1, pp. 181- 186 and vol.1, 13-16, 2005.

10. Addlesee, M., Jones, A., Livesey, F., Samaria, F., "ORL Active Floor". IEEE Personal Communications vol. 4(5), pp. 35-41, 1997.

11. Want, R., Hopper, A., Falcão, V., Gibbons, J. "The Active Badge Location System." ACM Transactions on Information Systems, pp. 91-102, 1992.
12. Patel, S. N., Reynolds, M. S., Abowd, G. D., "Detecting human movement by differential air pressure sensing in HVAC system ductwork: An exploration in infrastructure mediated sensing". Proceedings of the International Conference on Pervasive Computing (Pervasive 2008), Springer LNCS 5013, pp. 1-18, 2008.

13. Kotanen, M. Hannikainen, H. Leppakoski, T. D. Hamalainen, "Experiments on local positioning with Bluetooth," Proc. IEEE Int. Conf. Inf. Technol.: Comput. Commun., pp. 297-303, 2003.

14. Ni,L.M. Liu,Y. Lau, Y.C, Patil, A.P., "LANDMARC: Indoor location sensing using active RFID," Wireless Netw., vol. 10, no. 6, pp. 701-710, Nov. 2004.

15. Bahl, P., Padmanabhan, V.N., "RADAR: An inbuilding RF-based user Location and tracking system", Proc. IEEE INFOCOM 2000, vol. 2, pp. 775-784, 2000.

16. Hightower, J.,Want, R., Borriello, G., "Spot ON: An indoor 3D location sensing technology based on RF signal strength," Univ. Washington, Seattle, Tech. Rep. UW CSE 2000-02-02, 2000.

17. Wettergren, A., "ZigBee in Industry". Bachelor Thesis Performed in Computer Engineering, Linkoping Institute of Technology, Sweden, 2007.

18. Zigbee Alliance, "HomeToys interview", http://hometoys.com/emagazine.php?art_id=340, 2004.

19. Li, B., Kam, J., Lui, J., Dempster, A.G., "Use of directional information in wireless lan based indoor positioning.", Symp. on GPS/GNSS(IGNSS), 2007.

20. Tadakamadla, S.. "Indoor Local Positioning System for ZigBee, Based On RSSI". Master of Science thesis, Mid Sweden University, Sweden, 2006. 